\title{
STRUCTURE OF DECRYSTALLIZED COTTON PREPARED IN FIBER FORM BY ALKALI AND ACRYLONITRILE TREATMENTS
}

\author{
By Asako Hirai, Masao Hosono and Waichiro Tsuji* \\ (Institute for Chemical Research, Kyoto University, \\ Uji, Kyoto-Fu 611, Japan)
}

\begin{abstract}
Synopsis
The moisture regain and the crystalline structure of partially cyanoethylated cotton fibers were examined in comparison with the results on the cotton fabrics. Cotton was treated with acrylonitrile at $20^{\circ} \mathrm{C}$ and $0^{\circ} \mathrm{C}$ after immersing in $18 \mathrm{wt} \% \mathrm{NaOH}$ aqueous solution at $15^{\circ} \mathrm{C}$ and $-5^{\circ} \mathrm{C}$, respectively. A greater effect of cyanoethyl residue to retain highly decrystallized state of the treated cotton fibers was observed in the cyanoethylation at $0^{\circ} \mathrm{C}$ and the fibers showed greater moisture regain. On the contrary, the effect of temperature on the treatment for cotton fabrics was not so sensitive as in the case of fibers. Such factors as textile structure in fabrics, temperature in alkali-pretreatment and cyanoethylation, and the rate of diffusion of acrylonitrile were considered as the causes of the discrepancy.
\end{abstract}

\section{Introduction}

In the previous papers, ${ }^{1,2,3}$ we have reported that the crystallinity of cotton fabrics was considerably decreased by an alkali-acrylonitrile treatment involving cyanoethylation and that accessibility of the fabric could be improved. The crystalline structure of cellulose molecules in the fabric was examined by $X$-ray and infrared spectra in connection with the moisture regain. ${ }^{3}$ However, the cyanoethylation as well as the alkali swelling involved in this treatment may be influenced by the textile structure of the sample since fabrics are composed of twisted yarns.

In the present work, the cyanoethylation by the alkali-acrylonitrile treatment is applied to cotton fibers as well as cotton fabrics. The moisture regain and the crystalline structure of cyanoethylated cotton fibers are examined in comparison with the results obtained from the cotton fabrics.

\section{Experimental}

Samples. Egyptian cotton fibers supplied by Unitika Co. were extracted in a Soxhlet extractor

\footnotetext{
* Present address: Mukogawa Women's University, Ikebiraki, Nishinomiya, Hyogo-Ken 663 , Japan.
}

with ethanol and then ether. Each extraction was carried out for $6 \mathrm{hrs}$. Then, they were boiled in $1 \%$ aqueous solution of sodium hydroxide for $10 \mathrm{hr}$, washed with distilled water, immersed in $1 \%$ acetic acid, washed with water again and then air-dried. As the fabric sample, scoured and bleached 40's cotton fabric (plain weave) supplied by Toyo Spinning Co. was used.

Alkali-Acrylonitrile Treatment. Cotton was treated with the following two methods. Method 1: Cotton was immersed in $18 \mathrm{wt} \%$ sodium hydroxide solution for $30 \mathrm{~min}$ at $15^{\circ} \mathrm{C}$ and then treated with acrylonitrile at $20^{\circ} \mathrm{C}$. Method 2: Cotton was immersed in $18 \mathrm{wt} \%$ sodium hydroxide solution for $60 \mathrm{~min}$ at $-5^{\circ} \mathrm{C}$ and then treated with acrylonitrile at $0^{\circ} \mathrm{C}$. Then the treated cottons were immersed in dilute acetic acid, washed with water and air-dried. The procedures were performed in the slack state unless otherwise noted.

Nitrogen analysis. Nitrogen contents $(\% N)$ of the treated samples were determined by the microKjeldahl method. The values were converted to the degree of cyanoethylation (D.C.) by the following equation.

$$
\text { D.C. }(\text { in mole } \%)=\frac{162 \times N}{42-1.59 \times N}
$$


Moisture Regain. After vacuum drying for $3 \mathrm{hr}$ at room temperature, samples were conditioned at $20^{\circ} \mathrm{C}$ and $65 \%$ R.H. until constant weights were reached, and then weighed. The samples were dried for $24 \mathrm{hr}$ in vacuo at $40^{\circ} \mathrm{C}$ and weighed. The moisture regain was calculated by the following equation.

$$
\text { Moisture regain }(\%)=\frac{W_{\mathrm{b}}-W_{\mathrm{a}}}{W_{0}} \times 100
$$

Here, $w_{\mathrm{b}}$ and $w_{\mathrm{a}}$ are the weights of the samples before and after drying, respectively. $W_{0}$ is the weight of the residual cellulose estimated by subtracting the increase in weight by cyanoethylation from $W_{a}$.

X-Ray Analysis. $\mathrm{X}$-ray analysis was made with use of bundled fibers fixed with $1 \%$ collodion. The equatorial scanning was made on a Rigaku Denki Geiger Flex wide-angle X-ray diffractometer with $\mathrm{Cu}-\mathrm{K} \alpha$ beam at $40 \mathrm{kV}$ and $15 \mathrm{~mA}$. The X-ray profiles thus obtained were resolved into Gaussian curves with a du Pont 310 curve resolver.

\section{Results}

Figure 1 shows the relation between the reaction time with acrylonitrile and the degree of cyanoethylation. Curves (a) and (b) denote the results by the method 1 for the cotton fibers and fabrics, respectively. Curves (c) and (e) denote the results by the method 2 for cotton fibers and fabrics, respectively. The rate of reaction for the fibers is greater than that for the fabrics. This will be attributed to much greater effective surface area of the cotton fibers than that of the fabrics consisting of twisted yarns. When the fabrics were treated at constant length by the method 1 , the reaction rates of the samples were much smaller than those of samples in the slack condition, as shown in Figure 1 [curve (d)] .

Figure 2 shows the relation between the moisture regain and the degree of cyanoethylation. It is seen that for samples cyanoethylated by the method 1 in fiber and fabric forms, the moisture regains are almost same, although the rates of reaction are different $[(O)$ and $(\triangle)$ in curve (a)]. On the contrary, it is found that the moisture regain of fiber samples prepared by the method 2 [curve (b)] is higher than that of fabric samples [curve (c)]. But the moisture regain of fabric

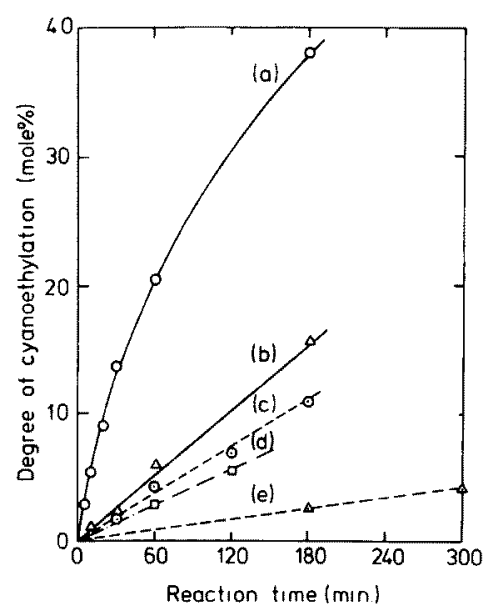

Fig. 1 Reaction time vs. degree of cyanoethylation. (a) cotton fiber and (b) cotton fabric, in slack state, and (d) cotton fabric, at constant length; immersed in $18 \mathrm{wt} \% \mathrm{NaOH}$ for $30 \mathrm{~min}$ at $15^{\circ} \mathrm{C}$ and then treated with acrylonitrile at $20^{\circ} \mathrm{C}$. (c) cotton fiber and (e) cotton fabric; immersed in $18 \mathrm{wt} \% \mathrm{NaOH}$ for $60 \mathrm{~min}$ at $-5^{\circ} \mathrm{C}$ and then treated with acrylonitrile at $0^{\circ} \mathrm{C}$.

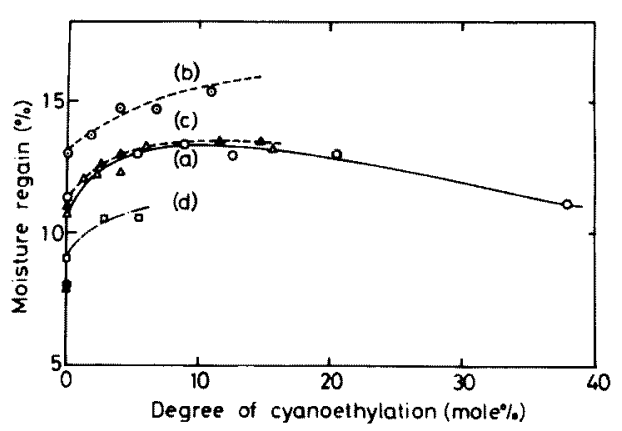

Fig. 2 Moisture regain vs. degree of cyanoethylation. (O) cotton fiber and $(\Delta)$ cotton fabric, in slack state, and ( $\square$ ) cotton fabric at constant length; immersed in $18 \mathrm{wt} \% \mathrm{NaOH}$ for $30 \mathrm{~min}$ at $15^{\circ} \mathrm{C}$ and then treated with acrylonitrile at $20^{\circ} \mathrm{C}$. ( $\left.\odot\right)$ cotton fiber and $(\Delta)$ cotton fabric, in slack state; immersed in $18 \mathrm{wt} \% \mathrm{NaOH}$ for $60 \mathrm{~min}$ at $-5^{\circ} \mathrm{C}$ and then treated with acrylonitrile at $0^{\circ} \mathrm{C}$. Filled circle and filled triangle at zero degree of cyanoethylation represent the values for original cotton fiber and fabric, respectively. 
samples prepared by the method 2 is nearly same to that of fabric samples prepared by the method 1 . When the fabrics were treated at a constant length by the method 1, moisture regain of the samples is much smaller than those of the samples treated in the slack conditions, as shown in Figure 2 [curve (d)].

Figure 3 shows X-ray equatorial scannings for fiber samples treated by the method 1 . The figure evidences that when cotton fibers are treated only with alkali solutions (mercerized cotton), the presence of crystalline form of cellulose $I$ is detected in addition to that of cellulose II, but the crystalline form of the alkali-acrylonitrile treated

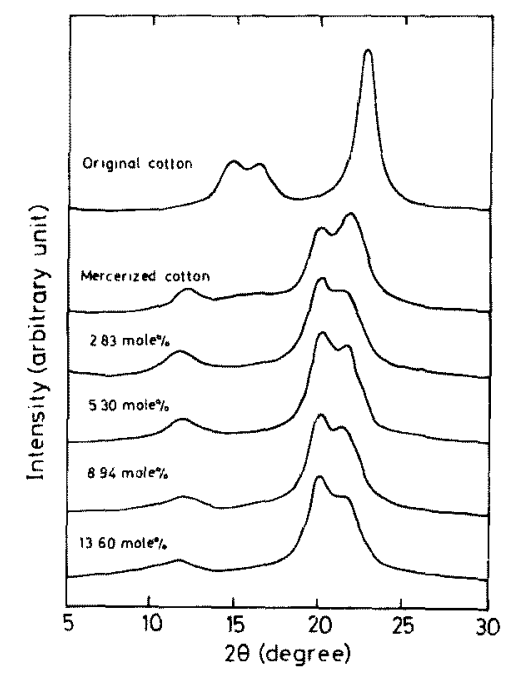

Fig. 3 X-ray diffractograms of cotton fibers immersed in $18 \mathrm{wt} \% \mathrm{NaOH}$ for $30 \mathrm{~min}$ at $15^{\circ} \mathrm{C}$ and then treated with acrylonitrile at $20^{\circ} \mathrm{C}$.

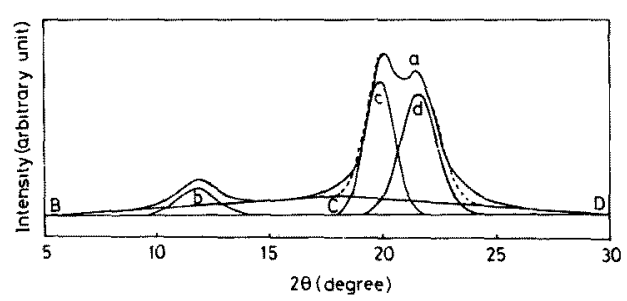

Fig. 4 Wide angle $X$-ray scattering pattern of cotton fiber treated under the same conditions as that for Fig. 3 (CN content, 5.30 mole\%). Curve a; experimental curve. Curves $b, c$, and $d$; resolved curves. Dotted curves; composite curve. fibers is exclusively that of cellulose II. However, as reported previously, ${ }^{3}$ when the same alkaliacrylonitrile treatment was applied for cotton fabrics, e.g. to an extent of cyanoethylation of 4.04 mole $\%$, the presence of the crystalline form of cellulose I was evidenced by the X-ray diffraction from the crystal planes (101) and (10T), although such evidence was not obtained for a sample with a degree of cyanoethylation of 5.93 mole\%. The X-ray diffraction profiles were resolved into Gaussian curves by means of a curve resolver in order to elucidate the crystalline structure of samples treated in the fiber form in more detail. Figure 4 illustrates a result for the sample with $\mathrm{CN}$ content of 5.30 mole\%. Here, curve $\mathrm{a}$ is the experimental profile and the straight line $\overline{\mathrm{BD}}$ indicates its baseline. The amorphous scattering is assumed to correspond to the area BCD. Curves $b$, $c$ and $d$ are those of the resolved components, and the dotted line shows the composite curve of the component curves.

Lattice spacings determined from the peak positions of the resolved curves are plotted against the degree of cyanoethylation in Figure 5. The open circles and triangles denote the results for fibers and fabrics, respectively. Here the result for

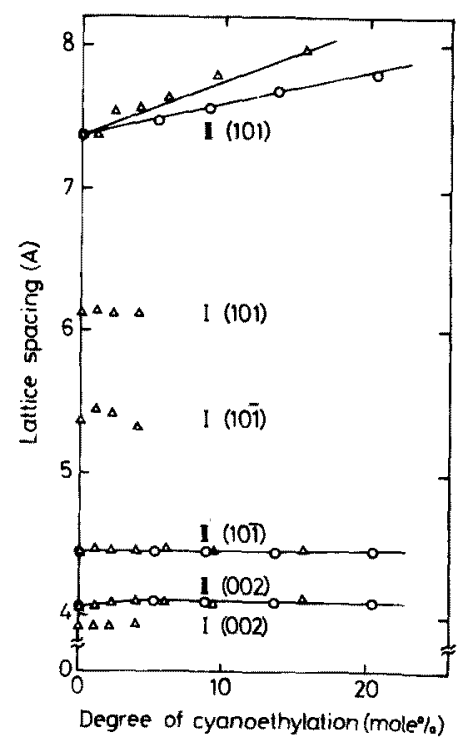

Fig. 5 Lattice spacing vs. degree of cyanoethylation. $(O)$ cotton fiber and $(\Delta)$ cotton fabric; immersed in $18 \mathrm{wt} \% \mathrm{NaOH}$ for $30 \mathrm{~min}$ at $15^{\circ} \mathrm{C}$ and then treated with acrylonitrile at $20^{\circ} \mathrm{C}$. 
fabrics was quoted from the previous report. ${ }^{3}$ The lattice spacing of (101) plane of cellulose II increases with increasing cyanoethyl content, while those of $(10 \overline{1})$ and $(002)$ planes remain nearly unchanged.

Figure 6 shows the X-ray equatorial scannings

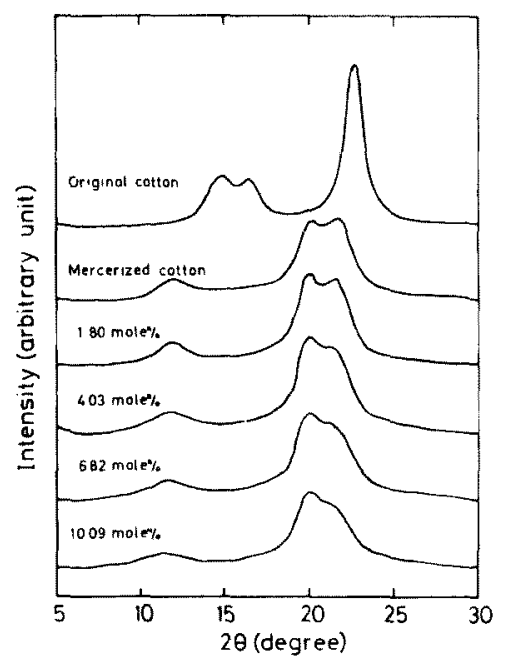

Fig. 6 X-ray diffractograms of cotton fibers immersed in $18 \mathrm{wt} \% \mathrm{NaOH}$ for $60 \mathrm{~min}$ at $-5^{\circ} \mathrm{C}$ and then treated with acrylonitrile at $0^{\circ} \mathrm{C}$.

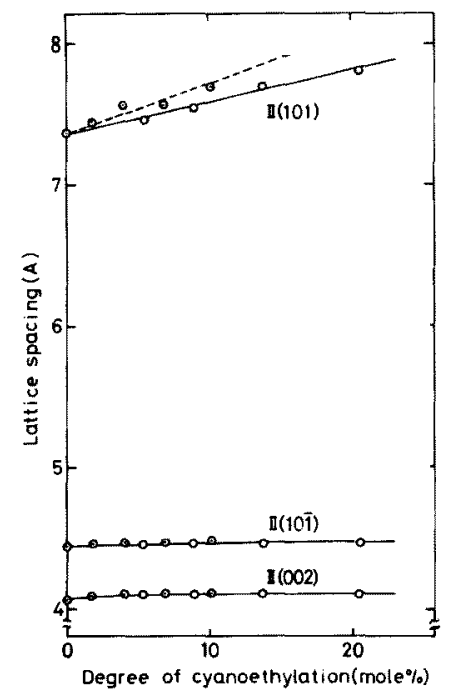

Fig. 7 Lattice spacing vs. degree of cyanoethylation. ( $\odot$ ) cotton fibers; immersed in $18 \mathrm{wt} \%$ $\mathrm{NaOH}$ for $60 \mathrm{~min}$ at $-5^{\circ} \mathrm{C}$ and then treated with acrylonitrile at $0^{\circ} \mathrm{C} .(O)$ cotton fibers; quoted from Fig. 5. for cotton fibers prepared by the method 2 . The crystalline form of the alkali-treated fibers (mercerized cotton) is completely cellulose II. From the resolved curves we determined the lattice spacings shown in Figure 7. In Figure 7, symbols $(O)$ and $(\odot)$ denote the results for fibers prepared by the method 1 and those for fibers prepared by the method 2 , respectively. Increase of lattice spacing of (101) plane denoted with $(\odot)$ is somewhat greater than that of samples denoted with $(O)$. Integral breadth of the samples prepared by the methods 1 and 2 is shown in Figure 8 . The increase of integral breadth of the (101) and (002)

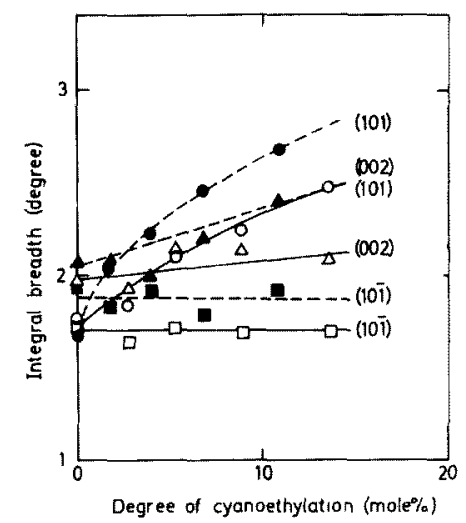

Fig. 8 Integral breadth of resolved curves of cotton fibers. (O) (101) plane, ( $\square)(10 \overline{1})$ plane, and $(\Delta)(002)$ plane of samples prepared by method 1. (O) (101) plane, (U)

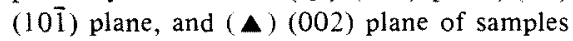
prepared by method 2 .

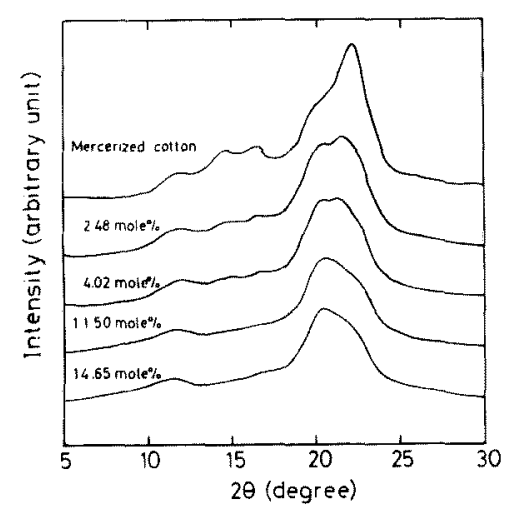

Fig. 9 X-ray diffractograms of cotton fabrics immersed in $18 \mathrm{wt} \% \mathrm{NaOH}$ for $60 \mathrm{~min}$ at $-5^{\circ} \mathrm{C}$ and then treated with acrylonitrile at $0^{\circ} \mathrm{C}$. 
reflections for the samples prepared by the method 2 is greater than that of the samples prepared by the method 1. As is well known, the integral breadth is associated with lattice perfection as well as crystallite dimensions.

From the results mentioned above, it was confirmed that greater decrystallization effect

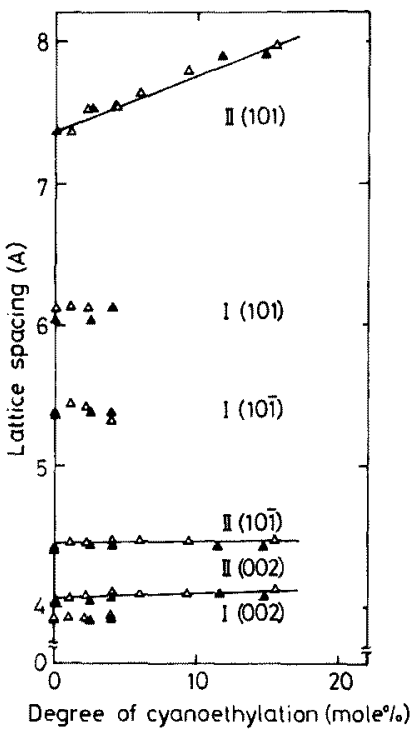

Fig. 10 Lattice spacing vs. degree of cyanoethylation. ( $\triangle$ ) cotton fabrics prepared by method 1. (A) cotton fabrics prepared by method 2 .

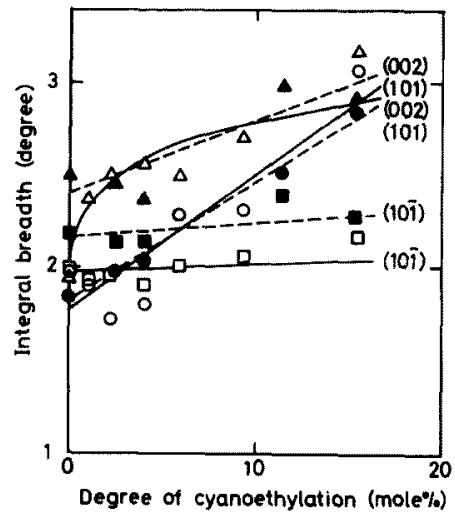

Fig. 11 Integral breadth of resolved curves of treated cotton fabrics. (O) (101) plane, $(\square)$ $(10 \overline{1})$ plane and $(\triangle)(002)$ plane of samples prepared by method 1. (O) (101) plane,

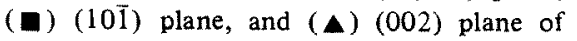
samples prepared by method 2 . associated with greater moisture regain was obtained in the fiber samples treated by the method 2. On the other hand, X-ray diffractograms of fabric samples prepared by the method 2 were similar to those of fabric samples prepared by the method 1 [see Figure 9]. In this case, the crystalline form of cellulose I was also detected in addition to that of cellulose II for some samples of a few mole \% cyanoethylation. As shown in Figures 10 and 11 , there is no marked difference in the crystalline structure between fabric samples prepared by the methods 1 and 2 .

\section{Discussion}

The textile structure composed of twisted yarns restricts swelling when the fabric is immersed in sodium hydroxide solution. As a result, each swollen fiber in the fabric is supposed to be under pressure or tension. Tension or pressure within fabrics may be responsible for the restricted mercerization as compared with yarn and fiber shown by Jeffries. ${ }^{4}$ In fact, when the fabrics were treated at constant length by the method 1 , the reaction rate and the moisture regain of the samples were much smaller than those of the samples treated in the slack condition, as shown in Figures 1 and 2 . It is clear that both the velocity of diffusion of acrylonitrile into fabrics and the effective surface area of fabrics are small compared with those of fibers in the slack state owing to the restraining influence of fabric construction. Consequently, the distribution of cyanoethyl residues will be nonuniform and hence the effect of cyanoethyl residues to retain the highly decrystallized state of treated cotton will be decreased. On the other hand, the fibers swell freely under the alkali-treatment. Accordingly it is expected, in the case of cotton fibers, that both the velocity of penetration of acrylonitrile into fibers and the effective surface area are larger than those in fabrics.

(a) Cyanoethylation at $20^{\circ} \mathrm{C}$ (method 1$)$.

The reaction rate at $20^{\circ} \mathrm{C}$ may be more rapid than the rate of diffusion of acrylonitrile, so that the cyanoethylation may take place mainly on the fiber surface and the distribution of cyanoethyl groups may be nonuniform. Therefore, the cyanoethylation by the method 1 yields products having nonuniform distribution of cyanoethyl groups for 
both fibers and fabrics. The difference in the effect of nonuniformity of cyanoethyl groups on the accessibility of fiber and fabric samples treated by this method is hardly discernible from the moisture regain at the same degree of cyanoethylation (see Figure 2). However, it is presumed from Figure 5 that there is a difference in the nonuniformity of cyanoethylation between fibers and fabrics from the standpoint of crystalline structure.

(b) Cyanoethylation at $0^{\circ} \mathrm{C}$ (method 2$)$.

The permanent decrystallization effect of the alkali-acrylonitrile treatment is probably caused by the cyanoethyl groups introduced into cotton cellulose in the swollen state which prevent the recrystallization of cellulose molecules. Hence, more uniform distribution of cyanoethyl groups resulted from a slower rate of cyanoethylation and more sufficient penetration of acrylonitrile into fiber may be advantageous to obtain more pronounced decrystallization effect. Thus in order to suppress the reaction of cyanoethylation while keeping the swollen state, the alkali-acrylonitrile treatment was carried out at a lower temperature. It is noted that when cotton is immersed in alkali solution, different crystalline forms are produced depending on the immersing temperature. When cotton is treated with $18 \mathrm{wt} \% \mathrm{NaOH}$ below $5^{\circ} \mathrm{C}$, soda cellulose $\mathrm{V}$ is obtained, ${ }^{5}$ whereas soda cellulose $I$ is obtained when treated with $18 \mathrm{wt} \% \mathrm{NaOH}$ at $20^{\circ} \mathrm{C}$. The composition of soda cellulose $\mathrm{V}$ is $\mathrm{C}_{6} \mathrm{H}_{10} \mathrm{O}_{5} \cdot \mathrm{NaOH} \cdot 4.5-5 \mathrm{H}_{2} \mathrm{O}$ and that of soda cellulose $\mathrm{I}$ is $\mathrm{C}_{6} \mathrm{H}_{10} \mathrm{O}_{5} \cdot \mathrm{NaOH} \cdot 3 \mathrm{H}_{2} \mathrm{O}$. So the unit cell of soda cellulose $\mathrm{V}$ is larger than that of soda cellulose I. To obtain uniform distribution of cyanoethyl residues the soda cellulose $\mathrm{V}$ seems to be preferable to the soda cellulose I. Furthermore, the time of immersing in alkali solution also could affect the decrystallization. ${ }^{6}$ Namely, it was reported that the crystallinity decreased and the degree of substitution increased with increasing swelling time, and leveled off at $45 \mathrm{~min}$.

Taking these facts into account, cotton immersed in $18 \mathrm{wt} \% \mathrm{NaOH}$ for $60 \mathrm{~min}$ at $-5^{\circ} \mathrm{C}$ was treated with acrylonitrile at $0^{\circ} \mathrm{C}(\operatorname{method} 2)$. In the case of fabrics, the influence of temperature in alkali-pretreatment is small and the penetration of acrylonitrile into fabric is poor on account of fabric construction. Therefore, there is no marked difference in the accessibility and crystalline structure for fabric samples prepared by the methods 1 and 2.

On the contrary, in the case of fibers, the influence of temperature in the alkali-pretreatment was observed unequivocally; alkali-pretreatment for $60 \mathrm{~min}$ at $-5^{\circ} \mathrm{C}$ results in the decrystallization of cellulose more than the treatment for $30 \mathrm{~min}$ at $15^{\circ} \mathrm{C}$. Moreover, the reaction is slow because of low temperature and the penetration of acrylonitrile into fiber is better than the case for fabrics. Consequently the distribution of cyanoethyl residues in the fiber sample prepared by the method 2 may be more uniform compared to that in the fabric sample and also to that in the fiber sample prepared by the method 1 . Therefore the greater moisture regain was obtained in the fiber samples prepared by the method 2 . The effect of cyanoethyl group on the crystalline structure of treated fibers by the method 2 was greater than that of treated fibers by the method 1 .

Part of this work was reported at the 21 st Kobe Meeting of the Society of Polymer Science Japan, 1975.

\section{Acknowledgement}

The authors wish to thank Professors A. Nakajima and R. Kitamaru for their useful discussions. The $\mathrm{X}$-ray analysis in this work was performed in Kobe University, with the help of Dr. K. Nakamae.

\section{References}

1) W. Tsuji and S. Imai; Bull. Inst. Chem. Res. Kyoto Univ., 44, 183 (1966).

2) W. Tsuji, A. Hirai and M. Hosono; J. Appl. Polym. Sci. 20, 2837 (1976).

3) A. Hirai, W. Tsuji, R. Kitamaru and M. Hosono, J. Appl. Polym. Sci. 20, 3365 (1976).

4) R. Jeffries, J. G. Roberts and R. N. Robinson, Text. Res. J., 38, 234 (1968).

5) I. Sakurada and K. Noma, "Dai-Yuki Kagaku" 19 (Tennen-Kobunshi Kagobutsu I), Asakura, p 50 (1960).

6) H. Maeda, K. Matsumoto, S. Kikuchi and K. Uemura, Sen-i Gakkaishi, 11, 721 (1955). 